International Journal of Pharmaceutics \& Pharmacology

EDWASER

Available Online: https://ijpp.edwiserinternational.com

\title{
Genetic Basis and Novel Treatment Strategies in Parkinson's Disease - A Review
}

\author{
Machhindra D. Bochare ${ }^{1}$ and Nagare Santosh Gangadhar ${ }^{2 *}$ \\ ${ }^{1}$ Director, ANA Education and Consultant, Nashik, Maharashtra, India \\ ${ }^{2}$ Department of Pharmacology, Banaras Hindu University, Varanasi, U.P. India
}

Article info

Received 01 January 2021

Revised 26 January 2021

Available Online 01 February 2021

*Corresponding author: Nagare Santosh Gangadhar, Department of Pharmacology, Banaras Hindu University, Varanasi, U.P. India

\begin{abstract}
Parkinson's disease (PD) is the 2nd most common progressive neurodegenerative disorder after Alzheimer's disease. Approximately 60000 are diagnosed with Parkinson's disease each year and more than 10 million people are living with $P D . P D$ is a neurodegenerative disorder in addition to the causes of PD are so many, it's not caused by a single pathophysiologic disturbance. So many drugs are available to treat PD but all are only for symptomatic relief no one drug is a disease-modifying agent. Although so many targets are available for targeting the Synuclein alpha, mitochondrial oxidative stress, autophagy, targeting glial cell inflammation, targeting metal ion homeostasis. But till now no one drug is successful in targeting these targets. In this review, we have summarized the genetic basis and novel targets available for the disease-modifying strategy for PD.
\end{abstract}

Keywords: Parkinson's disease; Neurodegenerative disorder; Synuclein alpha; Autophagy

\section{Introduction}

\section{Genetic Basis of Parkinson Disease}

Mutation in PRKN, PINK1 and PARK7 (autosomal recessive)

(1) PRKN- Individuals with PD have 2 copies of mutated genes through autosomal recessive inheritance [1]. PRKN (location chromosome 6), provides instruction for the making of PARKIN protein and this parkin protein is responsible for the degradation of unwanted protein which is no longer involved in cell survival. Parkin also plays important role in the proper functioning of mitochondria, it protects the mtDNA from oxidative stress it also enhances the mitochondrial membrane potential and reducing the ROS production from neurons [2]. More than 100 mutations of PRKN identified comprised of insertion or deletion of one or more exon and point mutation that causes the change in the reading frame, premature termination of translation, and some mutations of nonsense type led to dysregulation of all above-mentioned functions.

(2) PINK1- Located on chromosome 1 it provides instruction for making the protein PTEN induced putative kinase. This protein is located in mitochondria and protects the mitochondria from cellular stress. Missense, frameshift, point, truncating types of mutations have been found. PINK1 with parkin with the help of other mitochondrial protein remove the damaged mitochondria from healthy mitochondria. These damaged organelles are engulfed by autophagosomes and through lysosome mitophagy takes place [3].

(3) PARK7- This gene responsible for encoding the DJ1 and this protein protects the neurons from oxidative stress, helps in the folding of new proteins, and refolding of damaged proteins. DJ1 is highly expressed in cells that require high energy in $\mathrm{PD}$ there is a decreased level of DJ1 mRNA and an increased level of extra oxidized DJ isoform [4]. DJ1 acts as a biomarker in PD. 


\section{PINK1 and Parkin Functional Pathway}

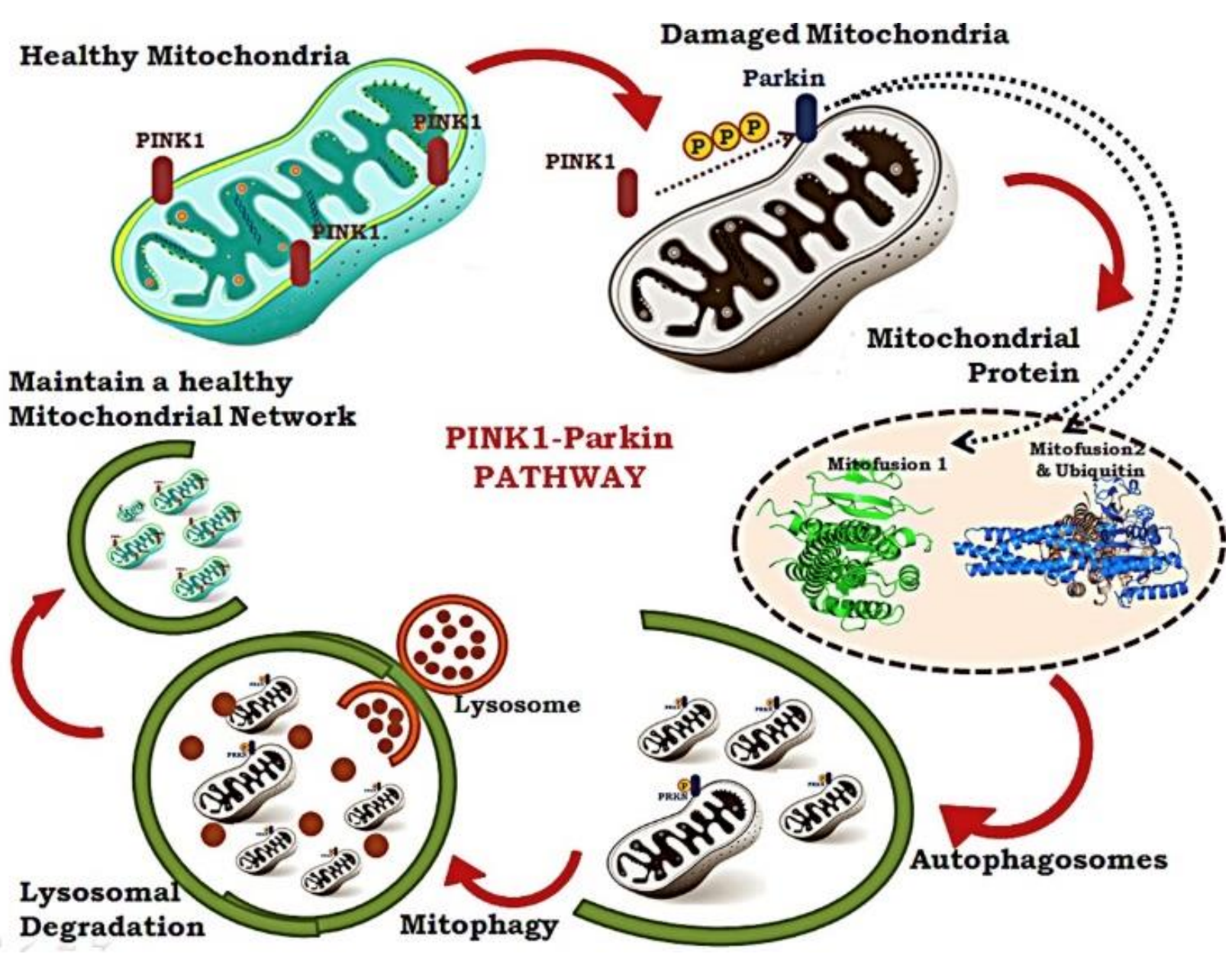

Figure 1: PINK1 and Parkin Functional Pathway.

LRRRK2 and SNCA Gene mutations (Autosomal dominance)

(1) LRRK2 (Leucine-rich repeat kinase 2) - It's located on chromosome 12 and belongs to the family ROCO Protein complex. The mutation increases kinase activity and neurodegeneration is kinase dependent [5]. LRRK2 mutants were shown to interact with Fas Ligand and then combines with adapter proteins to form a Fasassociated death domain which activates the caspases 8 which leads to neuronal degeneration [6]. LRRK2 also plays important role in regulation of protein translation, neurites morphogenesis, dynamics of the cytoskeletal and intracellular trafficking, synapse formation. These findings of kinase-dependent neurodegeneration support drug makers to generate LRRK2 kinase inhibitor as a therapeutic strategy for PD [7]. Pathologically more than $80 \%$ of autopsy examined cases show the presence of LRRK2 with Lewy bodies. LRRK2 level appears high in striatum, cortex, and cerebellum [8]. LRRK2 is a large protein of 2527 amino acid [9]. Lewy bodies are the most widely spread pathology in LRRK2 parkinsonism which are restricted to the brainstem, cortex, and limbic system. LRRK2 Provides instructions for protein dardarin and dardarin is important for cell functioning.

(2) SNCA - SNCA gene located on chromosome 4 which codes protein alpha-synuclein. Alpha-synuclein protein is located on the presynaptic terminal and is involved in supplying the synaptic vesicles containing dopamine to presynaptic terminals and the release of dopamine. This alpha-synuclein is involved in apoptosis suppression in dopaminergic neurons by downregulating the protein kinase $\mathrm{C}$ activity. It has been shown that alpha-synuclein stops the proteolytic cascade by downregulation of protein kinase delta expression [10]. It also involved in the regulation of glucose level, promotes the sensitive factor attachment to the protein receptor complex, acts as a molecular chaperone, maintenance of PUFAs level, Neuronal 
differentiation, regulation of dopamine biosynthesis, modulating vesicle trafficking. Alpha-synuclein chaperone activity depends on both the $\mathrm{N}$ and $\mathrm{C}$ terminal. $\mathrm{N}$ terminal domain responsible for interaction with substrate and $\mathrm{C}$ terminal domain carries out solubilization of that complex. Out of Alpha, Beta, Gamma synuclein [11] beta synuclein expressed most and gamma synuclein [12] is the least but in PD pathology alpha-synuclein is involved because the other synuclein is not the part of Lewy bodies [13].

\section{Novel Treatment Strategy for Parkinson Disease}

Targeting alpha synuclein aggregation - Alphasynuclein aggregation is the most common pathologic event in PD. Through 4 ways the disadvantageous effect of alpha-synuclein can be controlled.

(1) Reducing alpha-synuclein synthesis

(2) Preventing accumulation by enhancing degradation

(3) Inhibiting protein misfolding and aggregation

(4) Blocking cell to cell transmission

(1) Reducing alpha-synuclein synthesis- Mutation in the alpha-synuclein gene leads to PD. So, to reduce the level of alpha-synuclein level it's necessary to target these genes. This reduction is achieved by RNA interference by using a gene silencing mechanism to target alpha-synuclein mRNA levels [14]. In addition to this rodent's models demonstrated that antisense oligonucleotide safely reduced the level of alphasynuclein [15]. It's also reported that using a viral vector in rat plus non - human primate $\mathrm{SN}$ correspondent to the nigrostriatal system results in a $90 \%$ reduction of alphasynuclein [16].

(2) Preventing accumulation by enhancing degradationFor the degradation of abnormal alpha-synuclein novel approaches are like increasing autophagic clearance. Glucocerebrosidase pathway degrades the alphasynuclein but mutation in GBA leads to accumulation of alpha-synuclein, GBA also stabilizes alpha-synuclein oligomers [17].

(3) Inhibiting protein misfolding and aggregation -Heat and shock protein act as molecular chaperones Inhibiting protein misfolding and aggregation and promotes the correct folding of polypeptide chains so that there will no protein aggregation [18]. The interesting point of investigation is How exactly aggregation takes place despite the presence of chaperone proteins quality control system. In advanced pathology of PD, HSPs may also get trapped into the aggregates leads to a reduction in the availability of molecular chaperones. Another approach to preventing aggregation is through reducing the $\mathrm{C}$ terminal truncation which makes the alpha-synuclein protein prone to aggregation [19]. Oligomer modulator called Anle $\quad 138 \mathrm{~b} \quad[3-(1,3$ benzodioxol-5yl)-5-(3bromophenyl)-1H-pyrazole] inhibits the formation as well as accumulation of alpha-synuclein [20].

(4) Blocking cell to cell transmission- The recent findings that 14-3-3 can reduce the cell-to-cell transmission and toxicity associated with alphasynuclein [21]. Antibodies against $\mathrm{C}$ terminal truncation passive immunization that targets CT region of alphasynuclein mThy alpha- transgenic mouse model [22].

\section{Targeting Mitochondrial Dysfunction and Oxidative Stress}

Various ways to target mitochondria to correct abnormality into normal functioning:

- Mitophagy activation to destroy the abnormal mitochondria.

- Increasing mitochondrial biogenesis.

Mitophagy activation to destroy the abnormal mitochondria-

(1) Parkin-dependent mitophagy- loss of function of parkin responsible for prominent mitochondrial pathology and loss of dopaminergic neurons [23]. This is the most common pathway of mitophagy [24]. PINK1/Parkin regulates Ub dependent mitophagy. PINK1 is a mitochondrial sensor, Parkin as a signal amplifier, ubiquitin chains as the signal effector [25].

(2) Parkin independent mitophagy- This type of mitophagy is independent of parkin protein, it's carried out by receptor-mediated and ubiquitin ligase mediated mitophagy [26].

(3) Receptor-mediated mitophagy- several receptors have LIR (LC3- Interacting region) which is responsible for binding to LC3 to induce mitophagy [27].

(4) Ubiquitin ligase mediated mitophagy- A novel E3 ligase ARIH was found to be involved in mitophagy independent of PINK1 [28]. Mitochondrial Ubiquitin ligase activator NF-Kb1 is another ubiquitin ligase E3 that can compensate for the loss of parkin/PINK1 in PD [29]. 
Increasing mitochondrial biogenesis-Coenzyme Q10 and creatine both drugs failed in clinical trials both were based on the fact that they increase mitochondrial bioenergetics [30]. Coenzyme Q10 is an electron carrier for complexes 1 and 2 of the mitochondrial chain and in addition to that, it's also a free radical scavenger [31].

Oxidative stress - Mitochondrial dysfunction occurs because of the ROS and failure of endogenous antioxidants leads to oxidative stress [32]. Preclinical studies of using mitochondria-targeted antioxidants like vitamin $\mathrm{E}$ and urate are going on [33].

\section{Targeting Neuroinflammation}

Neuroinflammation in PD is because of activation of glial cells results in the release of proinflammatory cytokines. For this reason, targeting microglia and inflammatory cytokines release by using drugs or neuroprotective substances. Pituitary adenylate cyclaseactivating peptide is an anti-inflammatory peptide [34] that regulates the pathway activated by Camp and also decreases the release of proinflammatory cytokines. Pioglitazone an antidiabetic was also found to reduce incidences of PD but it failed in a clinical trial [35].

\section{Targeting Intracellular Calcium Homeostasis}

There are pieces of evidence that increase calcium influx plays important role in the pathogenesis of PD [36]. Defects in the regulation of calcium comes from intracellular calcium stores may be in the ER and other related organelles [37-40].

\section{Conclusion}

Parkinson's disease causes are multifactorial amongst those causes mutations in the various genes are of major concern because all the genes that we have discussed in this review plays major role in maintaining the normal functioning of the neuronal cells. Mutations in these genes leads to disruption of normal functioning and becomes abnormal leads to Parkinson's disease. In addition to genetic basis of PD synuclein alpha, mitochondrial dysfunction, Neuronal inflammation sustains the PD further and makes it difficult to treat. So in order to develop gene therapy and disease modifying strategy for PD treatment understanding of genetic basis and existing pathways for targeting various abnormal proteins and organelles are of prime importance.

\section{Conflicts of Interests}

The authors declare no conflict of interest.

\section{Funding}

None declared.

\section{References}

1. Weyandt LL. Clinical Neuroscience (Foundation of Psychological and Neurodegenerative Disorders), $2^{\text {nd }}$ edition 2019, Routledge Vanderbilt Avenue New York, P-143,135,136.

2. Lianeri M, Dorszewska J. Mutation in PRKAN and SNCA genes important for the progress of Parkinson's Disease. Curr Genomics, 2013; 14: 506

3. Selvaraj S, Piramanayagam S. Impact of Gene Mutation in the development of Parkinson's Disease. J Genes Dis 2019; 6: 124-128.

4. Repici M, Giorgini F. DJ-1 in Parkinson's Disease: Clinical insights and Therapeutic Perspective. J Clin Med 2019: 3-4.

5. Dawson V. Genetic mutation in PD- LRRK2 Biology, Institute for cell engineering P: 6-7.

6. Lin CH, Tsai PI, Wu RM, et al. LRRK2 Parkinson's Disease: From animal models to the cellular mechanism. J Rev Neurosci 2011; 22: 3-4.

7. Padmanabham S, Fiske BK, Baptista MAS. The Michael J. Fox foundations strategies for accelerating translation of LRRK2 into therapies for PD. J Cells 2020: 1.

8. Dachsel JC, Farrer MJ. LRRK2 Parkinson's Disease. J Neurological Review 2010: 542-547.

9. Li JQ, Tan L, Yu JT. The role of the LRRK2 gene in PD. J Molecular Degenerat.

10. Emamzadeh FT. Alpha-synuclein structure, function and interaction. J Med Sci 2016: 3-4.

11. Lavedan C, Leroy E, Torres R, et al. Genomic Organization and Expression of the human Beta Synuclein Gene (SNCB). J Genomics 174.

12. Lavedan C, Leroy E, Deheja A, et al. Identification, Localization, and characterization of Human Gamma Synuclein. J Human Genet 1998: 107-108.

13. Burre J. The synaptic function of Alpha-Synuclein. 2015: 69-70.

14. Fields CR, Vergniory NB, Martins RW. Targeting alpha-synuclein a therapy for PD. J Frontiers Mol Neurosci 2019: 6.

15. Alarcon-Aris D, Recasense A, Galofre M, et al. Selective alpha-Synuclein knockdown in monoamine neurons by intranasal oligonucleotide delivery: Potential therapy for PD. J Mol Ther 550566.

16. Gorbutyuk OS, Li S, Nash K, et al. In vivo RNAi mediated Alpha-Synuclein Silencing induces Nigrostriatal Degeneration. J Mol Ther 1450-1455. 
17. Ellis JM, Fell MJ. Current approaches to the treatment of PD. Bioorg Med Chem Ltd 2017: 7.

18. Klucken J, Shin Y, Masliah E, et al. HSP70 Reduces Alpha-synuclein aggregation and toxicity. J Biol Chem 279.

19. Li W, West N, Colla E, et al. Aggregation Promoting C- terminal truncation of alphasynuclein is a normal cellular process and is enhanced by the Familial Parkinson's disease Linked Mutations. J Proc Natl Acad Sci 2005: 102.

20. Wagner J, Ryazanov S, Leonov A, et al. A novel oligomer modulator for disease Modifying therapy of Neurodegenerative Diseases such as Prion and PD. J Acta Neuropathol 795-811.

21. Wang B, Underwood R, Kamath A, et al. 14-3-3 proteins reduce cell to cell transfer and propagation of Pathogenic alpha synuclein. J Neuroscience 2018: 8212-8230.

22. Games D, Valera E, Spencer B, et al. Anti-alpha synuclein immunotherapy reduces Alpha synuclein propagation in combined viral vector and transgenic model of synucleiopathy. J Neurosci 2014: 2.

23. Morais VA, Haddad D, Cruessarts K, et al. PINK1 los of function mutations affect mitochondrial complex 1 activity via Nduf A10 Ubiquinone uncoupling. J Science 2014: 204-206.

24. McWilliams TG, Mugit MM. PINK1 and Parkin emerging themes in mitochondrial homeostasis. J Current Opin Cell Biol 2017: 85-90.

25. Harper JW, Ordureau A, Heo JM. Building and Decoding Ubiquitin chains for Mitophagy. J Nature Rev Mol Cell Biol 2018: 95-100.

26. Villa E, Marchetti S, Ricci JE. No Parkin Zone mitophagy without Parkin. J Trends Cell Biol 2018: 883-885.

27. Liu 1, Sakakibara K, Chen Q, et al. Receptormediated Mitophagy In Yeast and Mammalian systems. J Cell Res 2014: 788-791.

28. Wong YC, Holzbaur EL. Temporal Dynamics of PARK2/Parkin and OPTN/ Optineurin during the mitophagy of damaged mitochondria. J Autophagy 2015: 423-424.
29. Liu J, Liu W, Li R, et al. Mitophagy in Parkinson's Disease: From Pathogenesis to Treatment. J Cells 2019: 3-4.

30. Kalia LV, Kalia SK, Lang AE. Disease modifying strategies for Parkinson's Disease. Movement Disorders 2015; 30: 1442-1450.

31. Beal MF. Bioenergetic Approaches for Neuroprotection in Parkinson's Disease. J Ann Neurl 2003: S39-S47.

32. Lewis VJ. Oxidative stress and Parkinson's Disease, J Frontiers Neuroanat 2015: 2.

33. Jin H, Kanthasamy A, Ghosh A, et al. Mitochondria Targeted Antioxidants for treatment of Parkinson's Disease: Preclinical and clinical outcomes. Biochim Biophys Acta 2004: 1283-1290.

34. Lee FS, Rajgopal R, Kin AH, et al. Activation of TrK Neurotrophin receptor signaling by Pituitary Adenylate cyclase - activating polypeptide. J Bio Chem 2002: 277.

35. Wong PS. Pioglitazone in early PD, A phase 2 multicentric, double-blind Randomized trial. J Lancet Neurol 2015: 5-8.

36. Zaichick SV, Kaitlyn M, Caraveo MG. The Role of Calcium Signalling in PD. Special collection: Neurodegeneration 521.

37. Caraveo G, Auluck PK, Whitsell L, et al. Calcineurin determines toxic versus beneficial responses to alpha-synuclein. Proc Natl Accd Sci USA; E3544-E3552.

38. Buick MV, Sierra-Margo A, Alarcon-Gill J, et al. Novel Approaches for the treatment of Alzheimer's and Parkinson's Disease. J Mol Sci. 2019: 15.

39. Jahangir MA, Anand C, Muheem A, Gilani SJ, Taleuzzaman M, Zafar A, Jaffer M, Verma S, Barkat MA. Nano Phytomedicine Based Delivery System for CNS Disease. Curr Drug Metabol 2020.

40. Muheem A, Jahangir MA, Jaiswal CP, Jafar M, Ahmad MZ, Ahmad J, Warsi MH. Recent patents, regulatory issues, and toxicity of nanoparticles in neuronal disorders. Curr Drug Metabol 2020.

This manuscript was peer-reviewed

Mode of Review: Single-blinded

Academic Editor: Dr. Abdul Hafeez.

Copyright: (C2021 Machhindra and Nagare. This article is distributed under the terms of the Creative Commons Attribution 4.0 International License (http://creativecommons.org/licenses/by/4.0/), which permits unrestricted use, distribution, and reproduction in any medium, provided you give appropriate credit to the original author(s) and the source, provide a link to the Creative Commons license, and indicate if changes were made. 